\title{
Exposure Start Day
}

National Cancer Institute

\section{Source}

National Cancer Institute. Exposure Start Day. NCI Thesaurus. Code C83289.

The day of the week an exposure began. 